\title{
Relationship of serum irisin levels and various demographic and biochemical factors in non-diabetic hemodialysis patients
}

\author{
Fereshteh Rafi'i1', Seyed Mohsen Hosaini², Banafsheh Yalameha ${ }^{3}$, Hamid Nasri ${ }^{*}$ \\ ${ }^{1}$ Department of Internal Medicine, Isfahan, University of Medical Sciences, Isfahan, Iran \\ ${ }^{2}$ Department of Epidemiology and Statistics, Faculty of Health, Isfahan University of Medical Sciences, Isfahan, Iran \\ ${ }^{3}$ Nickan Research Institute, Isfahan, Iran
}

\section{Correspondence to: \\ Hamid Nasri, Email; \\ hamidnasri@med.mui.ac.ir \\ Received: 5 Sep. 2020 \\ Accepted: 18 Dec. 2020 \\ ePublished: 29 Dec. 2020}

Keywords: Irisin, Hemodialysis, Body mass index

\begin{abstract}
Introduction: Irisin is a myokine secreted from the skeletal muscle. Preliminary studies showed that the serum irisin level was decreased in patients with chronic kidney disease (CKD), particularly in hemodialysis patients.

Objectives: This study aimed to examine the relationship between serum irisin level and different demographic and biochemical factors in non-diabetic hemodialysis patients.

Patients and Methods: This study was conducted on 61 non-diabetic stable hemodialysis patients. Serum level of irisin was measured by ELISA method. Demographic information was recorded for all subjects. In addition, biochemical factors were assessed using standard methods.

Results: In this study serum irisin level was $8.15 \pm 4.24 \mathrm{ng} / \mathrm{mL}$. There were positive correlations of serum irisin with gender $(P=0.014)$ (higher serum value of irisin in males) and hemodialysis doses $(r=0.32, P=0.01)$. Additionally, a negative correlation of serum irisin with body mass index (BMI) was detected $(r=0.26$, $P=0.036)$.

Conclusion: The positive correlation of serum irisin with hemodialysis doses and a negative correlation of serum irisin with BMI requires further investigations in other patients with more patients.
\end{abstract}

Citation: Rafi' F, Hosaini SM, Yalameha BY, Nasri H. Relationship of serum irisin levels and various demographic and biochemical factors in non-diabetic hemodialysis patients. J Prev Epidemiol. 2020;5(2):e31. doi: 10.34172/jpe.2020.31.

\section{Introduction}

Chronic kidney disease (CKD) is a public health priority that affected $10-12 \%$ of the population (1). The advanced stage of CKD is the end-stage renal disease (ESRD) which leads to an irreversible reduction in renal function. ESRD may result in death if it is not treated by dialysis or kidney transplantation (2). The prevalence of metabolic imbalance and abnormal energy hemostasis in CKD patients is estimated to be $20 \%-80 \%$ (3$5)$. In addition, changing energy balance in these patients could lead to overweight, protein-energy waste, obesity, and increased mortality (6). It has been indicated that an active and dynamic lifestyle can play an important role in preventing CDK among different individuals through exercise and physical activity (7). In fact, muscle tissue produces various cytokines and myokines such as irisin and also regulates various physiological and metabolic pathways. Irisin is a newly discovered myokine that released into the circulation through cleavage of fibronectin type III domain containing

\section{Key point}

In a study on 61 non-diabetic stable hemodialysis patients, we found positive correlation of serum irisin with hemodialysis doses and a negative correlation of serum irisin with BMI.

protein 5 (FNDC5) $(8,9)$. Irisin regulates energy and metabolic homeostasis and improves body weight, glucose tolerance, and insulin resistance (10). Several studies have investigated irisin levels in various diseases such as type 2 diabetes mellitus, nonalcoholic fatty liver disease, cardiovascular diseases, metabolic syndrome, and CKD (11-14). Irisin is not excreted by the kidneys and its level is reduced in the advanced stage of CKD. Therefore, the level of irisin can be considered a substantial indicator of renal function (8). Furthermore, some studies have revealed that irisin level was lower in CKD patients with hemodialysis than normal individuals $(15,16)$.

Considering the importance of irisin level in chronic renal failure and the socio- 
economic and psychological burden, that hemodialysis imposes on patients, and the need to improve the quality of life in these patients, it seems necessary to conduct further studies in this field.

\section{Objectives}

The purpose of the present study is to investigate the relationship between serum irisin level and various demographic and biochemical factors in non-diabetic hemodialysis patients.

\section{Patients and Methods}

\section{Patients}

In this analytical cross-sectional, 61 subjects (35 men and 26 women) with ESRD under hemodialysis were referred to Noor hospital, Isfahan in 2015. Demographic information including hypertension, starting date of hemodialysis, number of dialysis sessions per week, dialysis duration, medication consumption, and body mass index (BMI) was recorded for all subjects. Inclusion criteria included patients without diabetes, chronic or acute infection, cancer, and muscular diseases, obvious ischemia detected by electrocardiography, and presence of heart failure as well as consumption of corticosteroids and immunosuppressive medications. The hemodialysis dose was calculated by multiplying the time of hemodialysis duration and the number of dialysis sessions per patient until the test was taken. Dialysis adequacy was also calculated by $\mathrm{Kt} / \mathrm{V}$.

\section{Laboratory assessments}

Five $\mathrm{ml}$ of venous blood samples were taken from all patients after an 8-hour fast and pre-hemodialysis for three consecutive days, and then the samples were immediately transferred to the laboratory. Complete blood count was assessed and blood serum was separated. Serum irisin was measured using an ELISA kit (EASTBIOPHARM, Hangzhou Eastbiopharm Co. Ltd. China) according to manufacturers' instructions. Levels of alkaline phosphatase, triglyceride, total cholesterol, C-reactive protein, albumin, calcium, phosphorus, and vitamin D were evaluated by standard methods.

\section{Ethical issues}

The research followed the tenets of the Declaration of Helsinki. The institutional ethical committee at Isfahan University of Medical Sciences approved all study protocols. Accordingly, written informed consent was taken from all participants before any intervention. This study was extracted from M.D., thesis of Fereshteh Rafi'i at this university (Thesis \#394752).

\section{Statistical analysis}

SPSS software, version 20 (SPSS Inc., Chicago, IL) was applied to analyze all data. Data were expressed as mean \pm SD for continuous variables. To determine the relationship between variables, independent $t$ test, chi-square, analysis of variance (ANOVA) and Pearson's correlation coefficient tests were used. $P<0.05$ was considered statistically significant.

\section{Results}

Table 1 represents the results of correlations between various demographic data and irisin level using Pearson's correlation coefficient and independent t-test. According to Table 1, a significant correlation was observed between irisin level and gender $(P=0.01)$. Therefore, irisin level was significantly higher in men than in women (Figure 1). Furthermore, an inverse and significant difference between irisin level and BMI ( $P=0.03, \mathrm{r}=-0.26$, Table 1; Figure 2). Linear regression was used to determine the relationship between the irisin level, as a dependent variable, and the other variables, as independent variables. We found a positive and significant relationship only with the dialysis dose $(P=0.01, r=0.32$; Figure 3$)$. No meaningful difference was revealed between biochemical parameters and irisin levels ( $P>0.05$; Table 2).

\section{Discussion}

This study aimed to investigate the relationship between serum irisin levels and various demographic and biochemical parameters in non-diabetic hemodialysis

Table 1. Correlations of various demographic data with serum irisin

\begin{tabular}{llll}
\hline Variables & Mean \pm SD & r & $\boldsymbol{P}$ \\
\hline Gender & & & $0.01^{*}$ \\
Men & $9.29 \pm 4.15$ & - & \\
Women & $6.62 \pm 3.94$ & - & \\
\hline Age $(\mathrm{y})$ & $56.31 \pm 17.82$ & -0.17 & 19.0 \\
BMl $\left(\mathrm{kg} / \mathrm{m}^{2}\right)$ & $21.29 \pm 4.38$ & -0.26 & $0.03^{*}$ \\
\hline Dialysis dose & $728.91 \pm 890.95$ & 0.32 & $0.01^{*}$ \\
Dialysis adequacy & $1.36 \pm 0.37$ & -0.15 & 24.0 \\
\hline$* P<0.05$. & & & \\
\hline
\end{tabular}

$* P<0.05$.

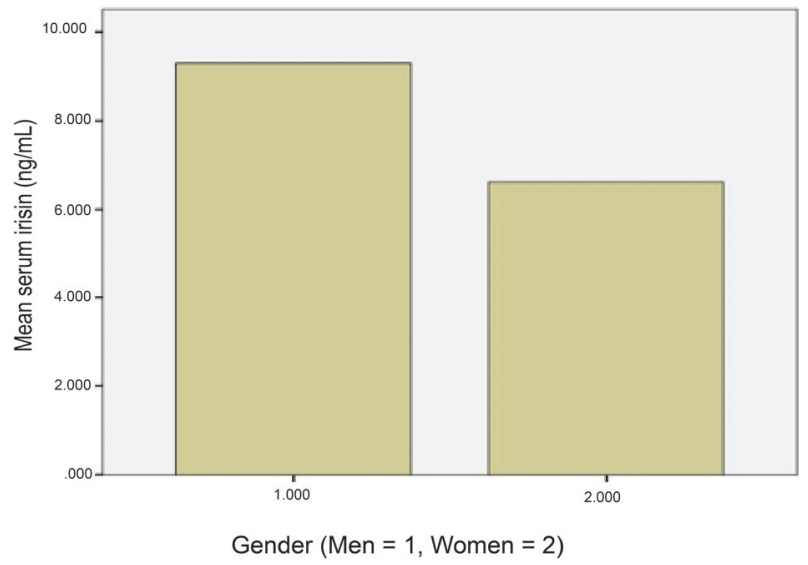

Figure 1. The correlation of irisin level and gender. 


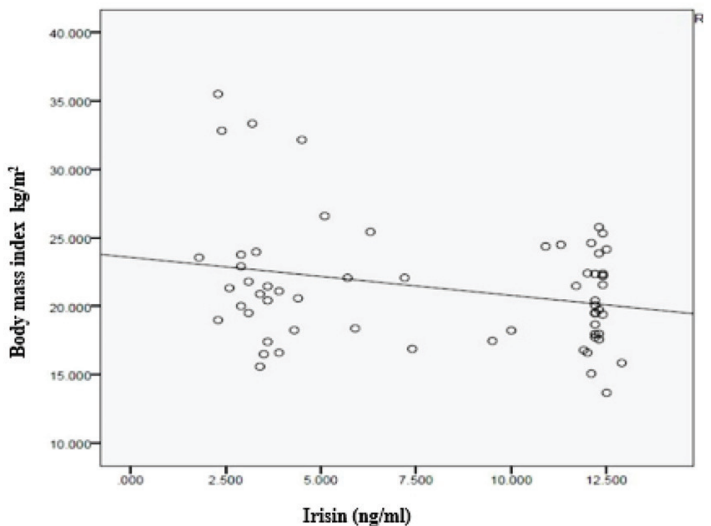

Figure 2. Inverse and significant correlation between BMI and irisin level $(P=0.03, \mathrm{r}=-0.26)$.

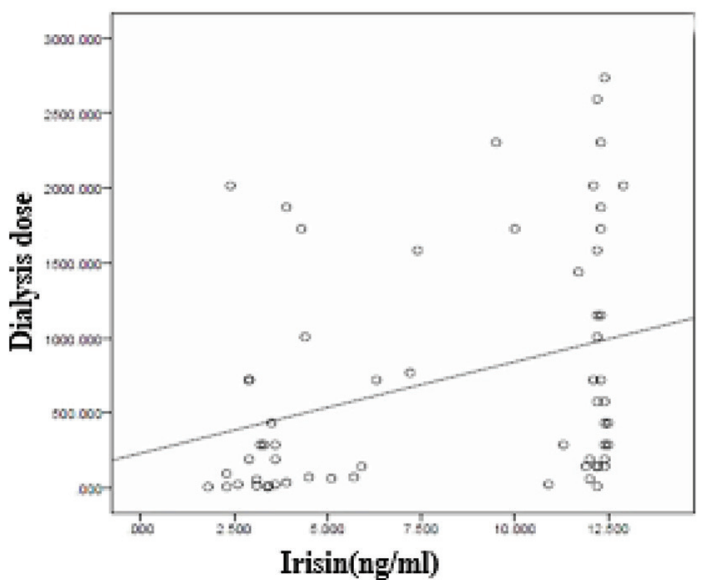

Figure 3. Positive and significant correlation between dialysis dose and irisin level $(P=0.01, \mathrm{r}=0.32)$.

patients in 2015. The findings indicated that serum irisin level has a significant relationship with gender that was higher in men.

In the study, conducted by Ebert et al on $532 \mathrm{CKD}$ patients from 1-5 stages, serum irisin levels were significantly greater in women than in men. In their study, only 169 out of $532 \mathrm{CKD}$ patients were in the $5^{\text {th }}$ stage and under hemodialysis (17). This stands as a possible reason for dissimilar findings with our findings.

Moreover, the present study showed an inverse relationship between serum irisin levels and BMI. A study conducted on 69 non-diabetic individuals with the mean BMI of $27.61 \pm 3.8 \mathrm{~kg} / \mathrm{m}^{2}$ reports that serum irisin level negatively correlates with BMI and fat mass (15). Choi et al found that serum levels of irisin have a negative correlation with BMI (18), which is consistent with the results of our study.

In a study involving three groups (group 1; patients with anorexia nervosa, group 2; those with normal weight, and group 3; patients with morbid obesity), serum irisin level in patients with obesity was higher than the other two groups, suggesting that irisin level positively correlated
Table 2. Correlation between various biochemical parameters and irisin levels

\begin{tabular}{llcc}
\hline Variables & Mean \pm SD & r & $\boldsymbol{P}$ \\
\hline Hemoglobin $(\mathrm{g} / \mathrm{dL})$ & $10.9 \pm 1.69$ & 0.06 & 0.62 \\
\hline Hematocrit $(\%)$ & $35.01 \pm 5.32$ & 0.05 & 0.68 \\
\hline Calcium $(\mathrm{mg} / \mathrm{dL})$ & $8.43 \pm 0.92$ & -0.1 & 0.44 \\
\hline Phosphorus $(\mathrm{mg} / \mathrm{dL})$ & $4.86 \pm 1.18$ & -0.14 & 0.26 \\
\hline Alkaline phosphatase $(\mathrm{U} / \mathrm{L})$ & $628.59 \pm 543.05$ & 0.05 & 0.67 \\
\hline Triglyceride $(\mathrm{mg} / \mathrm{dL})$ & $137.57 \pm 80.41$ & -0.24 & 0.057 \\
\hline Cholesterol $(\mathrm{mg} / \mathrm{dL})$ & $163.45 \pm 98.81$ & 0.13 & 0.3 \\
\hline Vitamin D $(\mathrm{ng} / \mathrm{mL})$ & $24.64 \pm 17.53$ & -0.02 & 0.86 \\
\hline CRP $(\mathrm{mg} / \mathrm{L})$ & $20.49 \pm 33.40$ & -0.2 & 0.11 \\
\hline Albumin $(\mathrm{g} / \mathrm{dL})$ & $3.39 \pm 0.56$ & 0.15 & 0.24 \\
\hline
\end{tabular}

with BMI $(13,14)$. In contrast, the study conducted by Wen et al on CKD patients indicated no correlation between serum irisin and BMI (16). This is not similar to the findings of the current study which can be possibly attributed to the higher BMI mean score $\left(23.06 \mathrm{~km} / \mathrm{m}^{2}\right)$ and the larger number of men than women (10 women, 28 men) which showed in the study by Wen et al (16).

In this study, a positive correlation between serum levels of irisin and dialysis dose was seen. The literature, however, does not seem to show a similar study conducted in this regard.

In our study, none of the biochemical variables including plasma hemoglobin, hematocrit, and serum calcium, phosphorus, alkaline phosphates, triglyceride, cholesterol, vitamin D and c-reactive protein showed a correlation with serum irisin levels. In the study by Cavalier et al serum irisin levels were measured after a dose of 100000 units of vitamin $\mathrm{D}$ in healthy volunteers, however, no relationship was found between vitamin $\mathrm{D}$ and irisin levels after the treatment (19).

In the study Ebert et al, 38 patients with chronic renal failure on stage five showed a positive correlation between serum irisin levels and HDL-c (17), while our study found no association between serum irisin levels and lipid profile. It should be noted, however, that in our study the total cholesterol level was measured as opposed to separate HDL-c and LDL-c values. This can probably be the cause for this inconsistency.

In the current study, the average cholesterol was calculated as $163.4 \mathrm{mg} / \mathrm{dL}$; this amount is $195.2 \mathrm{mg} /$ $\mathrm{dL}$ in the study by Ebert et al (17). Furthermore, in the study by Ebert et al, a positive correlation was reported between serum levels of irisin and plasma hemoglobin levels in patients (17), whereas in our study no correlation between these two variables and serin irisin was detected. The study conducted by Ebert et al involved 38 end-stage renal failure patients with $52.6 \%$ and $47.4 \%$ women and men respectively (17). However, our study included $42.6 \%$ of women. The duration of hemodialysis for patients in 
the study by Ebert et al was not reported. Thus, it may be due to differences in the duration of hemodialysis in these two studies which resulted in differences in plasma hemoglobin levels between the two studies.

\section{Conclusion}

In this study, we found that serum irisin level in hemodialysis patients correlates significantly with their gender such that it is more in men than women. It correlates negatively with BMI too. It correlated positively with dialysis dose too. Irisin is probably a weight-associated parameter whose shortage can lead to obesity. However, further studies are required as this is a novel issue. An important point to be taken into account in future research - possibly conducted with a larger sample size - is whether irisin is itself an agent or whether it plays the role of an epiphenomenon.

\section{Limitations of the study}

This study was conducted on 61 non-diabetic hemodialysis patients; wider research is required on all patients in other medical centers.

\section{Authors' contribution}

$\mathrm{HN}$ and FR were the principal investigators of the study. FR conducted the primary draft. SMH conducted the statistical analysis. BY conducted further edit. All authors read, revised, and approved the final manuscript.

\section{Conflicts of interest}

The authors declare no conflict of interest.

\section{Ethical considerations}

Ethical issues (including plagiarism, data fabrication, double publication) have been completely observed by the authors.

\section{Funding/Support}

This study was supported by a grant from Isfahan University of Medical Sciences.

\section{References}

1. Levin A, Tonelli M, Bonventre J, Coresh J, Donner JA, Fogo $A B$, et al. Global kidney health 2017 and beyond: a roadmap for closing gaps in care, research, and policy. Lancet. 2017;390:1888-917. doi: 10.1016/s0140-6736(17)30788-2.

2. Kusiak A, Dixon B, Shah S. Predicting survival time for kidney dialysis patients: a data mining approach. Comput Biol Med. 2005;35:311-27. doi: 10.1016/j.compbiomed.2004.02.004.

3. Kamimura MA, Draibe SA, Avesani CM, Canziani ME, Colugnati FA, Cuppari L. Resting energy expenditure and its determinants in hemodialysis patients. Eur J Clin Nutr. 2007;61:362-7. doi: 10.1038/sj.ejcn.1602516.
4. Brenner I, Brohart K. Weekly energy expenditure and quality of life in hemodialysis patients. CANNT J. 2008;18(4):36-40.

5. Avesani CM, Draibe SA, Kamimura MA, Dalboni MA, Colugnati FA, Cuppari L. Decreased resting energy expenditure in non-dialysed chronic kidney disease patients. Nephrol Dial Transplant. 2004;19:3091-7. doi: 10.1093/ndt/gfh547.

6. Levey AS, Coresh J. Chronic kidney disease. Lancet. 2012;379:165-80. doi: 10.1016/s0140-6736(11)60178-5.

7. Pedersen BK, Febbraio MA. Muscles, exercise and obesity: skeletal muscle as a secretory organ. Nat Rev Endocrinol. 2012;8:457-65. doi: 10.1038/nrendo.2012.49.

8. Panati K, Suneetha Y, Narala VR. Irisin/FNDC5--an updated review. Eur Rev Med Pharmacol Sci. 2016;20:689-97.

9. Kelly DP. Medicine. Irisin, light my fire. Science. 2012;336:423. doi: 10.1126/science.1221688.

10. Novelle MG, Contreras C, Romero-Picó A, López M, Diéguez C. Irisin, two years later. Int J Endocrinol. 2013;2013:746281. doi: 10.1155/2013/746281.

11. Polyzos SA, Kountouras J, Anastasilakis AD, Geladari EV, Mantzoros CS. Irisin in patients with nonalcoholic fatty liver disease. Metabolism. 2014;63:207-17. doi: 10.1016/j. metabol.2013.09.013.

12. Liu JJ, Liu S, Wong MD, Tan CS, Tavintharan S, Sum CF, et al. Relationship between circulating irisin, renal function and body composition in type 2 diabetes. J Diabetes Complications. 2014;28:208-13. doi: 10.1016/j.jdiacomp.2013.09.011.

13. Yang S, Xiao F, Pan L, Zhang H, Ma Z, Liu S, et al. Association of serum irisin and body composition with chronic kidney disease in obese Chinese adults: a cross-sectional study. BMC Nephrol. 2015;16:16. doi: 10.1186/s12882-015-0009-5.

14. Park KH, Zaichenko L, Brinkoetter M, Thakkar B, Sahin-Efe $A$, Joung $K E$, et al. Circulating irisin in relation to insulin resistance and the metabolic syndrome. J Clin Endocrinol Metab. 2013;98:4899-907. doi: 10.1210/jc.2013-2373.

15. Rodríguez-Carmona A, Pérez Fontán M, Sangiao Alvarellos S, García Falcón T, Pena Bello ML, López Muñiz A, et al. Serum levels of the adipomyokine irisin in patients with chronic kidney disease. Nefrologia. 2016;36:496-502. doi: 10.1016/j. nefro.2016.05.019.

16. Wen MS, Wang CY, Lin SL, Hung KC. Decrease in irisin in patients with chronic kidney disease. PLoS One. 2013;8:e64025. doi: 10.1371/journal.pone.0064025.

17. Ebert T, Focke D, Petroff D, Wurst U, Richter J, Bachmann A, et al. Serum levels of the myokine irisin in relation to metabolic and renal function. Eur J Endocrinol. 2014;170:501-6. doi: 10.1530/eje-13-1053.

18. Choi YK, Kim MK, Bae KH, Seo HA, Jeong JY, Lee WK, et al. Serum irisin levels in new-onset type 2 diabetes. Diabetes Res Clin Pract. 2013;100:96-101. doi: 10.1016/j. diabres.2013.01.007.

19. Cavalier É, Mismetti V, Souberbielle JC. Evaluation of circulating irisin levels in healthy young individuals after a single 100,000 IU vitamin D dose. Ann Endocrinol (Paris). 2014;75:162-4. doi: 10.1016/j.ando.2014.05.005. 